\title{
Estrategias argumentativas utilizadas por los estudiantes de la institución educativa Divino Niño de Tierra Grata (Chinú) en el análisis de hechos históricos culturales
}

\author{
Argumentative strategies used by students of the Educational Institution \\ Divino Niño de Tierra Grata (Chinú) in the analysis of historical cultural events
}

\section{RESUMEN}

En la enseñanza de las ciencias debe privilegiarse el desarrollo de la argumentación en pro de la construcción y comprensión de saberes en el aula. El presente estudio es un aporte a ese desarrollo y a la didáctica de la enseñanza de las ciencias a través del análisis del discurso de aula de los estudiantes de educación básica secundaria. Se reflexiona sobre la incidencia de las estrategias argumentativas y la calidad de los argumentos en la evolución conceptual de hechos históricos culturales (identidad, historia y cultura) valorando el modelo de la calidad

* El presente ensayo es producto del proyecto de investigación "Estrategias argumentativas utilizadas por los estudiantes al analizar hechos históricos culturales" gestionado en la Institución Educativa Divino Niño de Tierra Grata de Chinú (Córdoba, Colombia). Citar como Muñoz Arévalo, N. (2019). Estrategias argumentativas utilizadas por los estudiantes de la institución educativa Divino Niño de Tierra Grata (Chinú) en el análisis de hechos históricos culturales. Via Inveniendi et Iudicandi, 14(1), 49-66. Doi: https://doi.org/10.15332/ s1909-0528.2019.0001.02

** Licenciada en Ciencias Sociales de la Universidad de Córdoba. Magíster en Enseñanza de las Ciencias de la Universidad Autónoma de Manizales. Docente en la Institución Educativa Divino Niño de Tierra Grata de Chinú (Córdoba, Colombia). nemuare@hotmail.com. Orcid: https://orcid.org/0000-0001-7626-4318 
de los argumentos de Sampson y Clark (2009) y su relación con el modelo argumentativo de Candela (2006) y analizando sus implicaciones en la definición de contenidos que oscilan entre el conocimiento común y el científico.

Palabras clave: pensamiento crítico, conocimiento cualitativo, conocimiento científico, unidad didáctica (UD), argumentación, estrategias argumentativas, identidad, historia, cultura.

\section{Abstract}

In the teaching of science, the development of argumentation should be privileged, in favor of the construction and understanding of knowledge in the classroom. The present study is a contribution to their development and to the didactics of science teaching through the analysis of classroom discourse in secondary school students. In this, we reflect on the incidence of argumentative strategies and the quality of the arguments in the conceptual evolution of historical cultural events (Identity, History and Culture), assessing the quality model of the arguments of Sampson and Clark (2009) and their relationship with the argumentative model of Candela (2006) analyzing its implications in the definition of contents that oscillate between common knowledge and scientific knowledge.

Keywords: critical thinking, qualitative knowledge, scientific knowledge, didactic unit (UD), argumentation, argumentative strategies, identity, history, culture. 


\section{INTRODUCCIÓN}

El contexto actual exige la formación de estudiantes que adopten una actitud reflexiva y crítica frente a problemáticas que afectan a la sociedad. En este sentido, la argumentación debe estar presente en los procesos pedagógicos de aula. Diversidad de planteamientos contribuyen a aclarar el papel que esta juega en el desarrollo de la competencia comunicativa (Humbarita, 2015). Así, Candela plantea:

Por argumentación se entiende la articulación de intervenciones dentro de un discurso, con la intención de convencer a otro según un punto de vista. Implica, por tanto, que existen diferentes opiniones sobre algún tópico, por esto argumentar es presentar una postura con la conciencia de que existe una opinión implícita o explícita, diferente a la propia. (1991, p. 15)

Los aportes a la retórica de la Grecia antigua, la civilización romana y el pensamiento medieval fueron fundamentales en el campo argumentativo. Mientras tanto, en la Modernidad la retórica y con ello la argumentación fueron un poco relegadas porque las corrientes de pensamiento del siglo XVI y XVII privilegiaron la experimentación y la investigación cuantitativa y las ciencias encargadas de los problemas sociales aún no determinaban claramente sus objetos.

Desde el siglo XX hasta hoy se consolidan las disciplinas que buscan explicar fundamentalmente los fenómenos sociales, y con esto se presencia el resurgir de la argumentación. Así, Monzón afirma:

La recuperación y reformulación de la racionalidad condujo al surgimiento de movimientos que reviven el pensamiento retórico en autores como Perelman y Toulmin, quienes al mismo tiempo y sin haberse conocido publican en 1958 sendos libros sobre argumentación reviviendo algunos postulados de la retórica clásica. (2011, p. 3)

Las estrategias argumentativas y la calidad de los argumentos son importantes en los procesos comunicativos. Las primeras, según Candela (1991), se entienden como los 
recursos en los cuales se apoyan los argumentos y la segunda, según Sampson y Clark (2009), enfatiza en la estructura y complejidad de los conceptos.

En este estudio se privilegian las categorías argumentativas planteadas por Candela (1991): analogía, del conocimiento extraescolar al escolar y confrontación de punto de vista. Se establece además una relación con el modelo de la calidad de los argumentos planteado por Sampson y Clark (2009), conformado por las siguientes categorías: superficial, de sentido, de causa-consecuencia y abstracta.

A pesar de la importancia de la argumentación en la formación de los estudiantes se observan falencias en esta cuando se implementan en el aula técnicas como debates, exposiciones, foros, redacción de resúmenes, entre otras. Eso, aunado a la poca valoración que los estudiantes dan a la cultura tradicional, se convirtió en el interés del presente estudio, que buscó conocer las estrategias argumentativas utilizadas por los estudiantes del grado 9A de la Institución Educativa Divino Niño de Tierra Grata para analizar hechos históricos culturales en su entorno.

Entre los objetivos propuestos estaban identificar dichas estrategias, reconocer las situaciones de uso, señalar algunas estrategias argumentativas emergentes y diseñar una unidad didáctica. El estudio se concentra en los procesos pedagógicos del área de Ciencias Sociales porque allí es posible implementar nuevas estrategias metodológicas que evidencien la participación activa del estudiante a través de procesos argumentativos escritos y orales.

Entre las investigaciones relacionadas con estrategias argumentativas priman las desarrolladas en los niveles de educación básica primaria y superior. Se privilegiaron como antecedentes internacionales las siguientes:

a) "Del conocimiento extraescolar al conocimiento científico escolar". Es un estudio etnográfico en aulas de escuela primaria desarrollado por Candela (2006). El objetivo fue reconocer el papel que juega el discurso en la construcción social del conocimiento. En el aula y a través del discurso tal construcción es una tarea grupal en la cual se negocian significados y se construyen comprensiones compartidas (Edwards y Mercer, 1987), pero 
también se elaboran, argumentan y contraponen significados alternativos (Candela, 1991)

b) "Las estrategias argumentativas que regulan la interacción verbal en un aula de clase de Lengua Española”. Es una investigación realizada por Liseth Cornieles (2013) en la Universidad Simón Bolívar (Miranda, Venezuela). El objetivo general era analizar las estrategias argumentativas empleadas por el docente de Lengua Española para regular la interacción en el aula. La investigadora privilegia la clasificación que Cros (2003) hace de las estrategias argumentativas empleadas en el discurso docente para regular la interacción o establecer proximidad. Pretende así describir la forma como son empleadas tales estrategias por el docente. y en ese sentido observa la utilización de dos categorías, a saber: las estrategias basadas en la solidaridad y las basadas en la complicidad. Al respecto considera importante resaltar la necesidad de fortalecer la solidaridad entre las naciones para hacer frente a los problemas sociales actuales (Llano, Rengifo y Rojas, 2018).

c) "Razonamiento y argumentación en ciencias. Diferentes puntos de vista en el currículo oficial”. Realizada por Silvia García, José Manuel Domínguez y Eugenio García-Rodeja (2002), su objetivo fue evaluar el currículo oficial de Argentina para determinar si fomenta el aprendizaje de las estrategias de razonamiento y argumentación en niveles de enseñanza no universitarios y en la formación de profesores.

En concordancia con lo dispuesto en líneas anteriores es importante anotar que

uno de los más destacables enfoques en el uso de las tecnologías de la información y la comunicación (TIC), en el proceso de la enseñanza-aprendizaje, es poder implementar recursos educativos digitales (RED) que favorezcan la autonomía educativa en el estudiante y que le permitan al profesor centrarse en áreas de conocimiento más significativas y específicas. (Gómez, 2017, p. 149) 


\section{Población y Muestra}

El estudio se realizó con veintidós estudiantes del grado 9A de la Inedini (Institución Educativa Divino Niño de Tierra Grata, Chinú, Córdoba). Se tomó una muestra de la población al azar, conformada por seis estudiantes representados con las letras A, B, C, D, E y F.

\section{Metodología}

El estudio tiene un enfoque cualitativo insertado en un estudio de caso viabilizado a través de una unidad didáctica (UD). La metodología de investigación empleada es la explicada por el profesor Osorio (2018), lo que supuso recopilación y lectura sistemática de fuentes documentales de carácter primario y secundario. Dentro de las fuentes primarias se incluyen documentos oficiales (que comprenden informes de gestión gubernamental, leyes, decretos y resoluciones), crónicas noticiosas, comunicados de las organizaciones sociales y políticas, entre otros. Las secundarias incluyen artículos de revistas, análisis y columnas de opinión (Osorio, 2018).

\section{Procedimiento de obTención de Información}

La información se obtuvo en tres momentos: en el primero, se aplicó el instrumento de ideas previas (pretest); en el segundo, se introdujo la unidad didáctica, y el tercero, se midió el alcance o impacto de la propuesta.

\section{Procedimiento de ANÁlisis de INFORMaCión}

Para el análisis se procedió así: se seleccionó la información pertinente, se establecieron las categorías y subcategorías, se triangularon las respuestas por tendencias, se cruzaron los resultados y se obtuvieron las conclusiones. 
Lo anterior permitió precisar la evolución conceptual de acuerdo a las definiciones de algunos referentes teóricos como Lavacude (1997) y Tylor (1975), entre otros.

Tabla 1. Categorías conceptuales

Categoría

Identidad

\section{Definición}

La RAE (2017) define la identidad como el "conjunto de rasgos propios de un individuo o de una colectividad que los caracterizan frente a los demás”

Lavacude (1997) ve la historia como el análisis de los procesos humanos a través del tiempo; es decir, como un discurso que nos permite conocer los procesos políticos, económicos y socioculturales de las diversas sociedades en diversas épocas

Edward Tylor (1975) considera que la cultura es la totalidad de lo que aprenden los individuos como miembros de la sociedad; una forma de vida, un modo de pensar, de actuar y de sentir (Chinoy, 1980)

Fuente: elaboración propia.

Como ya se dijo, las estrategias argumentativas utilizadas por los estudiantes se analizaron según el modelo propuesto por Candela (2006), a saber: analogía, del conocimiento extraescolar al escolar y confrontación de punto de vista. Durante el desarrollo de las actividades fueron surgiendo estrategias argumentativas que no se hallaban dentro de dicho modelo. Entre estas estrategias emergentes están las de autoridad y ejemplo, fundamentadas en Weston (2003) (tabla 2).

Tabla 2. Estrategias argumentativas ejes de la investigación y estrategias emergentes

\begin{tabular}{lcl}
\multicolumn{1}{c}{ Categoría } & Abreviatura & \multicolumn{1}{c}{ Descripción } \\
\hline Analogía & ANG & $\begin{array}{l}\text { Afirmar una noción con base en la comparación de } \\
\text { circunstancias con otra }\end{array}$ \\
$\begin{array}{l}\text { Del contexto } \\
\text { extraescolar al } \\
\text { escolar }\end{array}$ & CEE & $\begin{array}{l}\text { En el proceso de construcción del conocimiento escolar } \\
\text { se emplean argumentos fundamentados en experiencias } \\
\text { cotidianas producto generalmente de la interacción social }\end{array}$ \\
\hline
\end{tabular}


Categoría

Confrontación de punto de vista

\section{Emergentes}

Autoridad

Ejemplo
Abreviatura

CPV

Lo que alguien dice se vincula en el debate con lo que otro expresa

Fuente: elaboración propia a partir de Candela, 2006; Weston, 2006.

El análisis del contenido se realizó a la luz del modelo de Schwarz (2008) considerado por Sampson y Clark (2009), el cual se fundamenta en la calidad de los argumentos como se muestra en la tabla 3.

Tabla 3. Categorías de análisis de contenido (calidad de los argumentos)

\begin{tabular}{|lcl}
\hline \multicolumn{1}{|c}{ Categoría } & Abreviatura & \multicolumn{1}{c}{ Descripción } \\
\hline Superficial & SU & Aquellos que no son precisos \\
\hline Abstracta & AB & Plantean razones con sentido lógico \\
\hline De sentido & DS & $\begin{array}{l}\text { Generalmente aceptados o que se basan en la propia } \\
\text { experiencia }\end{array}$ \\
\hline De consecuencia & DC & Hacen referencia a una relación causa-efecto \\
\hline
\end{tabular}

Fuente: elaboración propia con base en el marco de análisis de argumentos de Sampson y Clark, 2009.

En aras de presentar una información más completa se relacionaron las categorías de análisis de contenido con las de estrategias argumentativas teniendo en cuenta la definición de cada una (tabla4). 
Tabla 4. Relación entre las categorías de análisis de contenido y las de estrategias argumentativas

\section{Categoría de análisis de contenido}

Superficial (SU)

De sentido (DS)

De consecuencia (DC)

Abstracta $(\mathrm{AB})$

\section{Categoría argumentativa}

Solo opinión (OP)

Del contexto extraescolar al escolar (CEE)

Analogía (ANG)

Confrontación de punto de vista (CPV)

Autoridad (AU)

Ejemplo (EJ)

Fuente: elaboración propia.

Nota: las categorías argumentativas AU y EJ se trabajaron en el análisis paralelas a las del análisis de contenido.

\section{Resultados}

Para determinar la evolución conceptual y las estrategias se analizaron los argumentos emitidos por los estudiantes frente a los modelos antes anotados. Se puede afirmar que en la medida en que los argumentos planteados por los estudiantes y las estrategias argumentativas eran de mayor complejidad la evolución conceptual fue eminente.

A continuación se expone la tabla que permitió identificar la evolución conceptual y de las estrategias argumentativas de los estudiantes a partir de las categorías de análisis del contenido y estrategias argumentativas. 
Via Inveniendi et Iudicandi

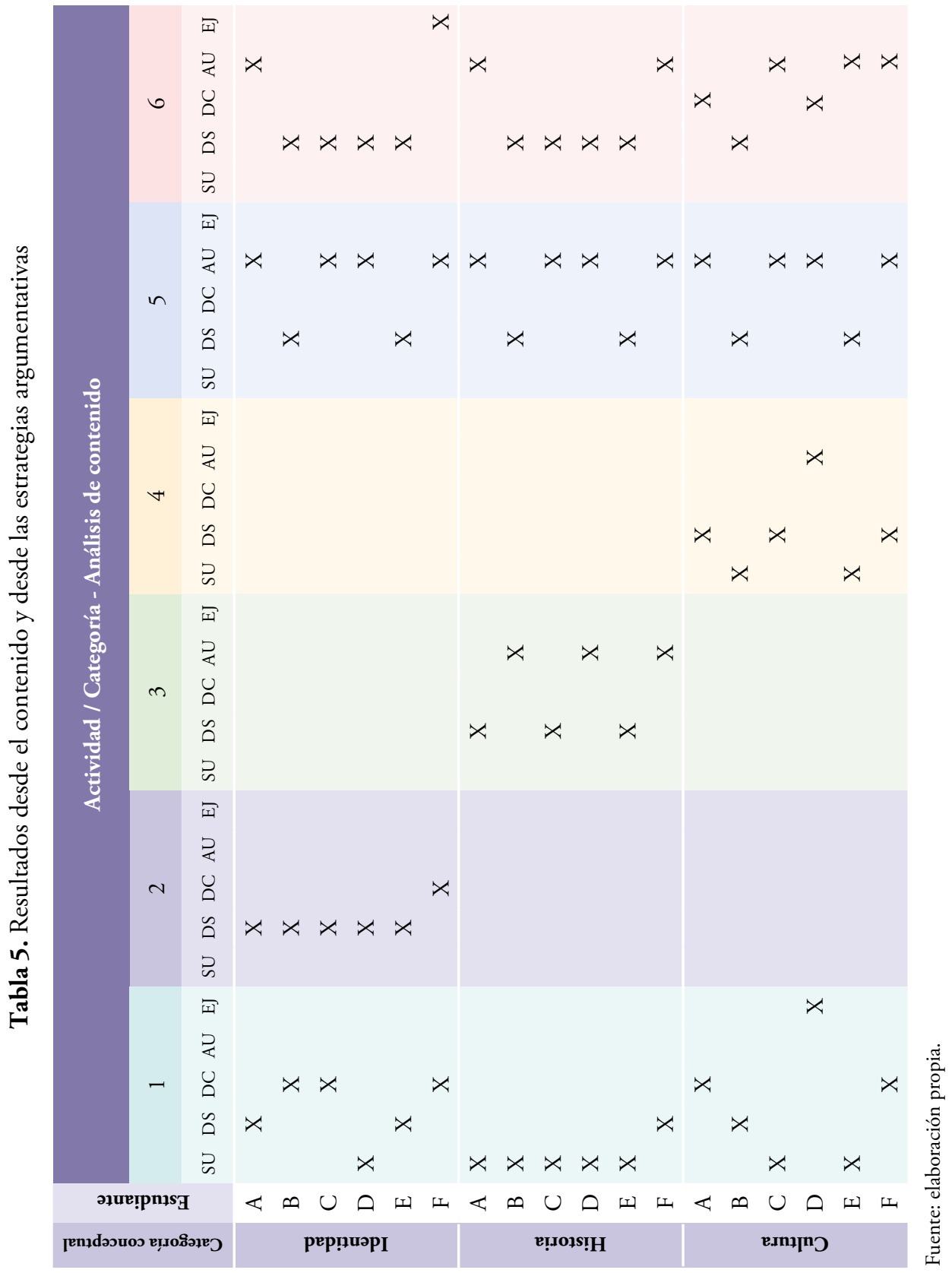


$\mathrm{Al}$ analizar la información expuesta en la tabla 6 se observa que al definir el concepto de identidad en el pretest los argumentos de los estudiantes variaron. Hubo estudiantes que expusieron solo su opinión, sin justificar sus ideas en conocimientos adquiridos dentro o fuera del aula o en fuentes documentales u orales, por los cual se consideran argumentos superficiales (SU) a la luz del modelo de Sampson y Clark (2009). Eso se observa en el caso del estudiante A, quien no utilizó ninguna estrategia argumentativa, sino que solamente emitió su opinión (OP); mientras que los estudiantes $\mathrm{B}, \mathrm{C}$ y $\mathrm{F}$ utilizaron el conocimiento de su entorno planteando argumentos de sentido (DS) apoyados en la estrategia argumentativa del contexto extraescolar al escolar (CEE). Lo anterior está en conexión directa con el hecho de que la afirmación de un concepto de derecho unívoco es particularmente compleja (Guarín y Aldana, 2016).

También se hallan estudiantes que argumentaron estableciendo comparaciones entre situaciones o aspectos alusivos a ciertos contextos teniendo en cuenta la relación de causa-efecto. Estos se pueden considerar argumentos de consecuencia (DC), que tienen cierto grado de credibilidad y se alejan así de saberes del común fundamentándose en dicha relación. Tales argumentos se pueden considerar también analógicos (ANG). Sin embargo, todos esos argumentos reflejan ideas populares y no evidencian el saber científico: les falta fundamentación en fuentes documentales producto de un trabajo riguroso que contribuya a la evolución conceptual.

En ese mismo sentido, se encontró que en el transcurso de la ejecución de la unidad didáctica (UD) la calidad de los argumentos reflejó avances significativos así: los estudiantes emitieron argumentos de sentido (DS) fruto del conocimiento de su contexto y de sus vivencias; estos son aceptados o validados en la medida en se basan en la experiencia; sin embargo, aunque tienen cierto asidero implícito en el conocimiento científico, aún no se pueden considerar abstractos.

Es preciso resaltar que la definición conceptual y la calidad de los argumentos evidencian ideas más completas, a diferencia de la superficialidad observada en la actividad 1. En la actividad 5 al definir el concepto en mención se observa que los estudiantes A, C, D y F emitieron argumentos abstractos fundamentados en fuentes orales producto de la salida pedagógica a la comunidad de Tierra Grata. Se nota 
así una evolución en la conceptualización del contenido: los estudiantes justificaron sus ideas en la información recolectada y utilizaron argumentos ubicables en las categorías emergentes de autoridad y ejemplo. Mientras tanto, los estudiantes $\mathrm{B}$ y C continuaron planteando argumentos de sentido (DS) basados en el conocimiento de su contexto inmediato, que es admitido por todos debido a su fundamentación en la experiencia. Por lo tanto, la estrategia argumentativa empleada en este último caso es del contexto extraescolar al escolar (CEE).

Finalmente, en la actividad 6, o postest, los estudiantes A y F fundamentaron su definición de identidad en fuentes documentales $\mathrm{u}$ orales, planteando argumentos abstractos (AB) y utilizando como estrategia la autoridad (AU). Los estudiantes B, $\mathrm{C}, \mathrm{D}$ y F, por su parte, utilizaron el conocimiento y la experiencia adquirida en su contexto inmediato emitiendo argumentos DS y apoyándolos en la relación entre el contexto extraescolar y el escolar (CEE).

En la definición del concepto de historia se observa que en la actividad 1, o pretest, el $100 \%$ de la muestra emitió una definición poco precisa, lo que evidencia la poca fundamentación argumentativa. Esto permite clasificar los argumentos emitidos como superficiales (SU), cuya característica principal es que reflejan la mera opinión personal, sin tener en cuenta los conocimientos del contexto o de fuentes documentales y orales. Es decir, en este caso los estudiantes no utilizaron ninguna estrategia argumentativa, solo emitieron su opinión (OP).

En la actividad 3 se observa que los estudiantes A, C y E utilizaron el conocimiento de su contexto para fundamentar su definición y construir el conocimiento escolar planteando argumentos que por su calidad son considerados de sentido (DS) — debe señalarse aquí que la experiencia extraescolar, según Candela (2006), permite reconstruir el conocimiento cotidiano para ir estableciendo el conocimiento científico escolar- Los otros estudiantes (B, D, F) fundamentaron su definición en fuentes documentales escritas y orales que emergen de conocimientos adquiridos de una monografía sobre el corregimiento de Tierra Grata que plasma los saberes de sus docentes y de habitantes de la comunidad. Los argumentos emitidos en este caso pueden ser considerados abstractos $(\mathrm{AB})$ pues se reflejan muchos tópicos implícitos en la definición del concepto por parte de algunos historiadores, que se alejan del 
saber popular o común (salvo la información recolectada en la comunidad). La estrategia argumentativa utilizada aquí se corresponde con la categoría emergente de autoridad (AU).

Los estudiantes B y E en la actividad 5 aprovecharon su experiencia cotidiana para fundamentar su definición planteando argumentos de sentido (DS) en los cuales se observa cómo los conocimientos previos de los educandos enriquecen sus procesos comunicativos. En cuanto a la estrategia argumentativa utilizada, se trata de la del contexto extraescolar al escolar (CEE). Los otros cuatro estudiantes (A, C, D y F) fundamentaron su definición en fuentes documentales planteando argumentos de carácter abstracto $(\mathrm{AB})$ correspondientes al uso de la estrategia de autoridad (AU).

En la actividad 6, o postest, se evidencian avances en la conceptualización y calidad de los argumentos. Un porcentaje representativo de estudiantes ofreció definiciones que se aproximan al saber científico, lo que se refleja en la complejidad de sus argumentos al definir el concepto en mención y en el uso de estrategias argumentativas de autoridad (AU) y ejemplo (EJ).

Al definir el concepto de cultura en la actividad 1, o pretest, se observa en la tabla que los estudiantes $\mathrm{C}$ y $\mathrm{E}$ fundamentaron su definición en sus propias ideas, sin basarse en otras fuentes. De allí que emitan argumentos incompletos que por su calidad pueden ser considerados superficiales (SU), correspondientes con la carencia de estrategia argumentativa alguna. Mientras tanto, los estudiantes A y F establecieron relaciones causa-efecto, comparando aspectos o situaciones y emitiendo argumentos DC relacionados con la estrategia argumentativa por analogía (ANG).

Solamente un estudiante (D) en su definición reflejó el uso de argumentos que por fundamentarse en fuentes orales pueden ser clasificados como abstractos (AB), relacionados con la estrategia argumentativa de autoridad (AU). El estudiante B, por su parte, emitió argumentos basados en su experiencia o conocimiento cotidiano del contexto, que pueden entenderse como argumentos de sentido (DS) correspondientes con la estrategia argumentativa del contexto extraescolar al escolar (CEE). 
En la actividad 4 se evidencia que los estudiantes B y E emitieron argumentos que pueden ser considerados como superficiales (SU) porque solo exponen su opinión. Por lo tanto, no soportaron sus ideas en estrategias argumentativas. Mientras tanto, A, C y F valoraron los conocimientos adquiridos en su contexto y se apoyaron en ello para fortalecer argumentos que según el modelo adoptado en este estudio se clasifican como de sentido (DS) y se corresponden con la estrategia argumentativa del contexto extraescolar al escolar (CEE). Además, los conceptos emitidos por A, C y F están implícitamente afincados en el conocimiento científico.

Un estudiante (D) en su definición deja claro que se apoya en evidencias que le permiten plantear tesis. Así, emite argumentos abstractos $(\mathrm{AB})$ que son aceptados como válidos por todos porque "tienen lógica". Esto enriquece la competencia comunicativa y se relaciona con el uso de la estrategia argumentativa de autoridad (AU).

En la actividad 5 al definir cultura se observa que los estudiantes A y D emitieron argumentos basados en la relación causa efecto (DC), que se relaciona con el uso de la analogía como estrategia argumentativa (ANG). Por su parte, los estudiantes $\mathrm{E}, \mathrm{F}$ y $\mathrm{C}$ realizaron la definición del concepto basándose en fuentes documentales y emitiendo argumentos abstractos $(\mathrm{AB})$ relacionados con el uso de la estrategia argumentativa de autoridad (AU).

En el postest afloran avances en la profundidad de la definición del referido concepto. Solamente un estudiante fundamentó su argumento en el conocimiento de su entorno y en su experiencia, que refleja la exposición de argumentos de sentido (DS) aceptados por todos. Pocos establecieron argumentos basados en la relación causa-efecto (DC). Predominaron los argumentos de autoridad (AU) basados en fuentes documentales, de tal forma que en la definición del concepto se observa una mayor aproximación al conocimiento científico, alejándose del saber común y de la mera opinión. 


\section{ConClusiones}

Los procesos argumentativos de los estudiantes de 9 A de la Inedini al iniciar la UD (unidad didáctica) eran débiles a la luz del modelo de calidad de los argumentos de Sampson y Clark (2009): prevalecían los argumentos superficiales caracterizados por expresar solo creencias propias no fundamentadas en fuentes escritas u orales, lo que influía en la ausencia del uso de estrategia argumentativa alguna para justificar afirmaciones, de modo que se quedaban en la mera opinión. Lo anterior se evidenció principalmente en la actividad inicial, específicamente en la definición simplista de historia.

En las primeras actividades también prevalecían argumentos de sentido (DS), que se fundamentan en la propia experiencia y el conocimiento del contexto, de modo que recurren a la estrategia argumentativa del contexto extraescolar al escolar (CEE) planteada por Candela (2006).

También fue notable el uso de la relación causa-efecto, que permitió a los estudiantes emitir argumentos de consecuencia (DC) relacionados con la estrategia argumentativa de la analogía (ANG).

A partir de la tercera actividad algunos estudiantes empezaron a plantear argumentos que por su calidad son considerados abstractos $(\mathrm{AB})$, caracterizados por la utilización de estrategias argumentativas emergentes (autoridad y ejemplificación), las cuales se analizaron según lo expuesto por Anthony Weston (2006).

Se observó que ningún estudiante utilizó la estrategia argumentativa de confrontación de punto de vista (CPV), lo que permite afirmar que es necesario seguir profundizando en el desarrollo de la argumentación ya que el debate de ideas no suele estar presente en la interacción comunicativa escolar.

Teniendo en cuenta lo anterior la evolución conceptual de los estudiantes fue notable: de una definición de los contenidos soportada en argumentos poco complejos basados más en el conocimiento común y la experiencia hacia definiciones más completas y próximas a los conceptos científicos. 
Se destaca así la importancia de la implementación de la unidad didáctica como herramienta metodológica pues contribuye a mejorar los procesos argumentativos. En efecto, en ella se pueden plasmar variadas actividades - como talleres, debates, exposiciones, mesas redondas, foros, ensayos, salidas pedagógicas y resúmenes— que brindan al estudiante la oportunidad de participar en la construcción de saberes.

De igual forma se pueden llevar al aula los problemas candentes y socialmente vivos, como el que motivó esta investigación: son propicios para planear e implementar estrategias y técnicas que permitan al estudiante desarrollar el pensamiento crítico, y dentro de este la argumentación, a través del uso de diversas estrategias como la analogía, el contexto extraescolar al escolar, la confrontación de punto de vista, la autoridad y el ejemplo, entre otras.

Por todo lo anterior esta investigación se convierte en un referente para que los docentes diseñen estudios relacionados con la argumentación identificando la situación en la que se hallan sus estudiantes en aras de implementar estrategias que permitan fortalecerla.

\section{REFERENCIAS}

Candela, A. (1991). Argumentación y conocimiento científico escolar. Infancia y Aprendizaje, 55(1), 13-28.

Candela, A. (2006). Del conocimiento extraescolar al conocimiento científico escolar. Investigación temática, 11(30), 797-820.

Chinoy, E. (1980). La sociedad: una introducción a la sociología. México: FCE.

Cornieles, L. (2013). Las estrategias argumentativas que regulan la interacción verbal en un aula de clase de lengua española. Paradigma, 34(2). Recuperado de https://goo.gl/ JJTZF9

Cros, A. (2003). Convencer en clase. Argumentación y discurso docente. Barcelona: Ariel. 
Edwards, D. y Mercer, N. (1987). Common knowledge. The development of understanding in the classroom. Londres: Methuen.

García, S., Domínguez, J. M. y García-Rodeja, E. (2002). Razonamiento y argumentación en ciencias. Diferentes puntos de vista en el currículo oficial. Enseñanza de las Ciencias, 20(2), 217-228.

Gómez, A. (2017). Elaboración del guion instruccional mediante la herramienta didáctica del recurso educativo digital. Via Inveniendi et Iudicandi, 12(2), 149-180. Doi: http:// dx.doi.org/10.15332/s1909-0528.2017.0002.06

Guarín, E. A. y Aldana, J. (2016). Estado jurisdiccional y bien común. Verba Iuris, 11(36), 13-26.

Humbarita, J. (2015). Derecho constitucional hispanoamericano frente a la realidad institucional, manifiesta divergencia. Revista Iusta, 2(43), 91-118. Recuperado de https://goo. $\mathrm{gl} / \mathrm{xaMDoh}$

Lavacude, K. (1997). Ciencias sociales. Bogotá: Santillana.

Llano V., Rengifo, R. y Rojas, L. (2018). Estado cosmopolita en América Latina. Revista Iusta, 1(48), 97-117. Doi: https://doi.org/10.15332/s1900-0448.2018.0048.04

Monzón, L. (2011). Argumentación: objeto olvidado para la investigación en México. Revista Electrónica de Investigación Educativa, 13(2), 41-54.

Osorio, R. (2018). La extradición y la cooperación internacional. Falta de justicia, legitimidad o incapacidad del Estado colombiano: su historia. Revista Iusta, 1(48), 179-198. Doi: https://doi.org/10.15332/s1900-0448.2018.0048.07

RAE (Real Academia Española) (2017). Identidad. En Diccionario de la lengua española. Recuperado de http://dle.rae.es/?id=KtmKMfe 
VIeI $\begin{aligned} & \text { Revista Virtual } \\ & \text { Via Inveniendi et Iudicandi }\end{aligned}$

Sampson, V. y Clark, D. (2009). The impact of collaboration on the outcomes of scientific argumentation. Science Education, 93, 448-484.

Tylor, E. (1975). La ciencia de la cultura. Barcelona: Anagrama.

Weston, A. (2006). Las claves de la argumentación. Barcelona: Ariel. 